\title{
CALCULATIONS OF CYLINDRICAL P-HOMOTOPY GROUPS
}

\author{
by R. AYALA, E. DOMÍNGUEZ and A. QUINTERO
}

(Received 1st February 1988)

\section{Introduction}

The definitions of the various proper homotopy groups correspond to three main geometrical ideas: sequences of spheres converging to a Freudenthal end (Brown groups); infinite cylinders giving the mobility of spheres towards a proper end (CerinSteenrod groups); sequences of spheres, each one movable to the next one following a proper end (Cech groups). The Brown and Cech groups have a rather complex structure and the calculations of these groups are very difficult (see [4]). The Cerin-Steenrod groups have a much simpler structure and this fact eases the computations.

These proper homotopy groups of a space $X$ are usually defined fixing one end of $X$ and missing the others. Therefore it seems interesting to define groups which give information of how the ends of $X$ are related to each other. In other words, the study of the mobility of spheres in $X$ from one end to another (see remark 2.2.(a)). In [1] we defined the cylindrical $p$-homotopy groups which are proper homotopy invariants associated to a given finite set of proper ends. In particular these groups generalize the Cerin-Steenrod groups. In this paper we work out some cylindrical p-homotopy groups and study the mobility of spheres in open surfaces.

In a subsequent paper we will prove that the cylindrical p-homotopy groups define proper homotopy functors with good properties as consequence of having proved that these groups are included in the general theory of cofibrations of Baues (see [2]). Also the cylindrical $p$-homotopy groups have a translation into the pro-homotopy category Ho(Pro-Top, Top) via the Edwards-Hastings embedding (see [8] and [12]). This viewpoint can be used to simplify some proofs (Proposition 1.1 and Proposition 1.3). We wish to thank T. Porter for his useful talks on this aspect of proper homotopy theory.

A proper map (p-map) is a continuous map $f: X \rightarrow Y$ such that $f^{-1}(K)$ is compact for each $K \subset Y$ closed and compact. Proper homotopy ( $p$-homotopy), proper homotopy equivalance, etc. are defined in the natural way.

$B^{n}$ and $S^{n-1}$ stand for the canonical ball and sphere in the Euclidean space $\mathbb{R}^{n}$; these spaces are based by $* \in S^{n-1}$. The unit interval is denoted by $I$.

Given a natural number $r, T[r]$ is the space obtained identifying the origins of $r$ copies of the half line $\mathbb{R}_{+}=[0, \infty)$. We assume that $T[r]$ is linearly embedded into $\mathbb{R}^{2}$ in 
such a way that the identification point is the origin of $\mathbb{R}^{2} . T[r, k]$ is the topological product $T[r] \times \mathbb{R}^{k}$. We identify $T[r]$ with $T[r, 0]$.

Given the topological spaces $X$ and $Y,[X, Y]$ is the set of homotopy classes of continuous maps and $[X, Y]_{p}$ is the set of $p$-homotopy classes of $p$-maps.

A base $T[r, k]$-tree on $X$ is a $p$-map $u: T[r, k] \rightarrow X$. Henceforth $T[r, k]$ is $T[r, k]$-based by the identity map. If $\left(Y, y_{0}\right)$ is compact, the product $Y \times T[r, k]$ is $T[r, k]$-based by $u_{0}(z)=\left(y_{0}, z\right)$. The set

$$
\left[S^{n} \times T[r, k], u_{0} ; X, u\right]_{p}
$$

of $p$-homotopy classes of $p$-maps preserving base trees, under the relation of $p$ homotopy relative to these base trees, is a group if $n \geqq 1$ and an abelian group if $n \geqq 2$. These groups are called the cylindrical $p$-homotopy groups and they are denoted by $\pi_{n}^{T}{ }^{[r, k]}(X, u)$ or, when there is no cause for confusion, by $\pi_{n}^{T[r, k]}(X)$. These groups can be generalized to pairs $(X, A)$ with $A \subset X$ closed and they are $p$-invariants. The basic facts on $\pi_{n}^{T}(-)$ are collected in [1]. When $r=1$ and $k=0, \pi_{n}^{T}(-)$ are the Črin-Steenrod groups (see $[5,1])$ which we denote by $\pi_{n}(X, u)$.

\section{The groups $\pi_{n}^{T[\mathrm{r}, \mathrm{k}]}(\pi[s, h])$}

If we consider $T[r, k]$ as an Euclidean set in $\mathbb{R}^{k+2}$ we can define

$$
S[r, k-1]=\{z \in T[r, k] ;|z|=1\}
$$

where " $\mid "$ is the Euclidean norm. This space is homeomorphic to the join $S^{k-1} *$ $\left\{p_{1}, \ldots, p_{r}\right\}$.

Proposition 1.1. There is a canonical bijection

$$
\Psi:[T[r, k] ; T[s, h]]_{p} \rightarrow[S[r, k-1] ; S[s, h-1]]
$$

Proof. By the Edwards-Hastings embedding theorem ([8, 6.2.7 $])$ the set $[T[r, k] ; T[s, h]]_{p}$ can be identified with the set $\Omega$ of (pro-Top, Top)-homotopy classes of (pro-Top, Top)-maps between the spaces $T[r, k]$ and $T[s, h]$ embedded in (proTop, Top). Now these spaces are contractible and their end neighbourhoods are $S[r, k-1] \times \mathbb{R}_{+}$and $S[s, h-1] \times \mathbb{R}_{+}$respectively; then $\Omega$ is in bijection with $[S[r, k-1] ; S[s, h-1]]$.

Remark 1.2. It will be useful to give an explicit description of $\Psi$. If

$$
E_{\delta}[r, k]=\{z \in T[r, k] ;|z|>\delta\}, \quad S_{\delta}[r, k-1]=\{z \in T[r, k] ;|z|=\delta\},
$$

given a $p$-map $f: T[r, k] \rightarrow T[s, h]$ we can find $\delta>0$ such that $f\left(E_{\delta}[r, k]\right) \subset E_{\delta}[s, h]$. By using the canonical retraction $\rho: E_{\delta}[s, h] \rightarrow S_{\delta}[s, h-1]$ we get a map $f: S_{\delta}[r, k-1] \rightarrow$ 
$S_{\delta}[s, h-1]$. If $f^{\prime}: S_{1}[r, k-1] \rightarrow S_{1}[s, h-1]$ is given by $f^{\prime}(z)=1 / \delta|f(\delta z)|$, we have $\Psi\left([f]_{p}\right)=\left[f^{\prime}\right]$.

Proposition 1.3. If $u: T[r, k] \rightarrow T[s, h]$ is a base tree and

$$
f_{u}: S[r, k-1] \rightarrow S[s, h-1]
$$

is a map whose homotopy class is $\Psi([u])$ there is a canonical isomorphism

$$
\Theta: \pi_{n}^{T r, k]}(T[s, h], u) \rightarrow \pi_{n}\left(\operatorname{Map}\left(S[r, k-1], S[s, h-1], f_{u}\right) .\right.
$$

Proof. As in the proof of Proposition 1.1, the Edwards-Hastings embedding theorem provides an isomorphism

$$
\Theta: \pi_{n}^{T[r, k]}(T[s, h], u) \rightarrow \pi_{n}^{S[r, k-1]}\left(S[s, h-1], f_{u}\right) .
$$

Now the result follows from the Duality Theorem $([11,6.2 .38])$.

\section{Corollary 1.4.}

(a) $\pi_{n}^{T[r, k]}(T[s], u)=0 \quad(k \geqq 1$ or $n \geqq 1)$.

(b) $\pi_{n}^{T[r, k]}(T[1, h], u)=0$ (compare with Theorem 4.1 ).

(c) $\pi_{n}^{T[r]}(T[s, h], u) \simeq \prod^{r} \pi_{n}\left(\bigvee^{s-1} S^{h}\right)$, where $\bigvee^{s-1} S^{h}$ is the wedge of $s-1$ copies of $S^{h}$.

(d) $\pi_{n}^{T[2, k]}(T[2, h], u) \simeq \pi_{n}\left(\operatorname{Map}\left(S^{k}, S^{h}\right), f_{u}\right)$.

\section{Corollary 1.5.}

(a) $T[r]$ has the same p-homotopy type as $T[s]$ if and only if $r=s$.

(b) If $k \geqq 1, T[r, k]$ and $T[s]$ have the same p-homotopy type if and only if $r=s=1$.

(c) If $h, k \geqq 1$ and $r, s \geqq 2, T[r, k]$ and $T[s, h]$ have the same p-homotopy type if and only if $r=s$ and $h=k$.

Remark 1.6. According to $1.4(\mathrm{~d})$ the calculations of $\pi_{n}^{T[2, k]}(T[2, h], u)$ rely on the calculations of the Hurewicz groups of the maps space $\operatorname{Map}\left(S^{k}, S^{h}\right)$. These groups have been studied in [9] and [10] and from these works we can state:

(a) $\pi_{1}^{T[2,2]}(T[2,2], u) \simeq \mathbb{Z} / 2 p \mathbb{Z}$ where $p$ is the degree of $f_{u}$. This proves that our groups depend on the tree $u$.

(b) If $h=1,3$ and $7, \pi_{n}^{T[2, k]}(T[2, h], u)$ does not depend on $u$. Moreover this group is isomorphic to $\pi_{n}\left(S^{h}\right) \oplus \pi_{n+k}\left(S^{h}\right)$.

(c) $\quad$ If $k \geqq 5, \pi_{1}^{T[2, k]}(T[2, k], u) \simeq \mathbb{Z} / 2 \mathbb{Z}$.

Proposition 1.7. Let $\varepsilon: T[r, k] \rightarrow T[s, h]$ by the p-map defined by 


$$
\varepsilon(z)=(0,0,|z|, 0, \ldots, 0) \in \mathbb{R}^{2} \times \mathbb{R}^{h} .
$$

If $G_{1}=\pi_{n}\left(\bigvee^{s-1} S^{h}\right)$ and $G_{2}=\pi_{k+n}\left(\bigvee^{s-1} S^{h}\right)$ are abelian groups, then $\pi_{n}^{T[r, k]}(T[s, h], \varepsilon) \simeq$ $\oplus^{-1}\left(G_{1} \oplus G_{2}\right)$.

Proof. Firstly the image of $\varepsilon$ by the bijection of Proposition 1.1 is the constant map *. By Proposition 1.3 and by the corresponding homotopy equivalences

$$
\pi_{n}^{T[r, k]}(T[s, h], \varepsilon) \simeq \pi_{n}\left(\operatorname{Map}\left(\bigvee^{r-1} S^{k}, \bigvee^{s-1} S^{h}\right), *\right)
$$

Now fixing a sphere $S^{k}$ in the wedge $\mathrm{V}^{r-1} S^{k}$ we get the cofibre sequence

$$
\mathrm{rin}^{\mathrm{-2}} S^{k} \rightarrow \stackrel{r-1}{\bigvee} S^{k} \rightarrow S^{k}
$$

which gives a weak fibration

$$
\operatorname{Map}\left(S^{k}, \bigvee^{s-1} S^{h}\right) \rightarrow \operatorname{Map}\left(\bigvee^{-1} S^{k}, \bigvee^{s-1} S^{h}\right) \rightarrow \operatorname{Map}\left(\bigvee^{r-2} S^{k}, \bigvee^{s-1} S^{h}\right)
$$

([13, II.8.8]). As the cofibre sequence admits an obvious coretraction, the homotopy exact sequence of (II) splits. Therefore (I) is isomorphic to

$$
\pi_{n}\left(\operatorname{Map}\left(S^{k}, \bigvee^{-1} S^{h}\right), *\right) \oplus \pi_{n}\left(\operatorname{Map}\left(\bigvee^{r-2} S^{k}, \bigvee^{s-1} S^{h}\right), *\right)
$$

Inductively we prove that $(\mathrm{I}) \simeq \bigoplus^{-1} H$, where

$$
H=\pi_{n}\left(\operatorname{Map}\left(S^{k}, \bigvee^{-1} S^{h}\right), *\right)
$$

Finally, it is proved in [9] that $H \simeq G$.

\section{A pull-back theorem}

In this section we fix a base tree $u: T[r] \rightarrow X$. Given $1 \leqq i \leqq r$ let $j_{i}: \mathbb{R}_{+} \rightarrow T[r]$ be the 
canonical embedding of $\mathbb{R}_{+}$as the $i$ th branch of $T[r]$ and $u_{i}=u \circ j_{i}$. The base point of $X$ will be $x_{0}=u(0)$.

Theorem 2.1. For each $1 \leqq i \leqq r$ let

$$
\rho_{i}: \underline{\pi}_{n}\left(X, u_{i}\right) \rightarrow \pi_{n}\left(X, x_{0}\right)
$$

the morphism defined by $\rho_{i}([f])=\left[f^{\prime}\right]$ where $f^{\prime}=f \mid S^{n} \times\{0\}$. If

$$
\left\{p_{i}: G \rightarrow \underline{\pi}_{n}\left(X, u_{i}\right) ; 1 \leqq i \leqq r\right\}
$$

is the pull-back of $\left\{\rho_{i}\right\}$ there is an epimorphism $\gamma: \pi_{n}^{T[r]}(X, u) \rightarrow G$ such that $p_{i} \circ \gamma=\omega_{i}$, where $\omega_{i}([f])=\left[f \circ j_{i}\right]$. Furthermore, if $r \geqq 2$ and $\pi_{n+1}\left(X, x_{0}\right)=0, \gamma$ is an isomorphism.

Proof. The morphism $\gamma$ is given by the pull-back universal property. We next prove that $\gamma$ is epimorphism.

Given $\left(g_{1}, g_{2}, \ldots, g_{r}\right) \in G$ we take a representative $f_{i}$ for each $g_{i} \in \underline{\pi}_{n}\left(X, u_{i}\right)$. The restrictions $f_{i} \mid S^{n} \times\{0\}(1 \leqq i \leqq r)$ define the same element $[h] \in \pi_{n}\left(X, x_{0}\right)$. Then there are homotopies $G_{i}$ relating $h$ and $f_{i} \mid S^{k} \times\{0\}$. The gluing of $G_{i}$ and $f_{i}$ provides [f] such that $\gamma[f]=\left(g_{1}, \ldots, g_{r}\right)$.

Assuming the extra hypotheses, if $\gamma[f]=0$ then $\omega_{i}[h]=0$ and we have $p$-maps $f_{i}: B^{n+1} \times \mathbb{R}_{+} \rightarrow X$ which extend $f_{i}=f \circ j_{i}(1 \leqq i \leqq r)$.

Now, if we regard $S^{n+1}$ as the gluing of two copies of $B^{n+1}, \pi_{n+1}\left(X, x_{0}\right)=0$ implies that

$$
\widetilde{f}_{1}\left|B^{n+1} \times\{0\} \cup \widetilde{f}_{i}\right| B^{n+1} \times\{0\}: S^{n+1} \rightarrow X(2 \leqq i \leqq r)
$$

extends to $f_{1 i}: B^{n+2} \rightarrow X$. It is obvious that $\left\{f_{i}, f_{1 i}\right\}$ gives a $p$-extension $f: B^{n+1} \times T[r] \rightarrow$ $X$ of $f$.

Remarks 2.2. (a) It is easy to find $(X, u)$ such that $\pi_{n+1}(X) \neq 0$ and $\gamma$ is not injective. For instance let $X$ be constructed by gluing two copies of $B^{2} \times \mathbb{R}_{+}$along $S^{1} \times\{0\}$. If $u: \mathbb{R} \rightarrow X$ is the natural map $t \rightarrow(*, t)$ it is not hard to prove that $S^{1} \times \mathbb{R} \rightarrow X$ defines a non-trivial element of $\pi_{1}^{T[2]}(X, u)$. But $\underline{\pi}_{1}\left(X, u_{i}\right)$ is obviously trivial for $i=1,2$.

(b) Also we can find spaces with $\pi_{n+1}(X) \neq 0$ and $\gamma$ is an isomorphism. For instance $X=S^{2} \times \mathbb{R}$ and $u(t)=(*, t)$. By Corollary $4.2, \pi_{1}\left(X, u_{i}\right)=\pi_{1}^{T[2]}(X, u)=1$.

(c) Note that Theorem 2.1 remains true for $n=0$.

Corollary 2.3. If $\pi_{n}\left(X, x_{0}\right)=0=\pi_{n+1}\left(X, x_{0}\right)$ then

$$
\pi_{n}^{T[r]}(X, u) \simeq \prod_{i=1}^{r} \underline{\pi}_{n}\left(X, u_{i}\right)
$$


Corollary 2.4. Let $\left\{\delta_{i}: \underline{\pi}_{n}\left(X, u_{i}\right) \rightarrow H\right\}$ be the push-out of

$$
\left\{\omega_{i}: \pi_{n}^{T[r]}(X, u) \rightarrow \underline{\pi}_{n}\left(X, u_{i}\right)\right\}
$$

and suppose that $\pi_{n+1}\left(X, x_{0}\right)=0$. Then $\left\{\omega_{i} ; 1 \leqq i \leqq r\right\}$ induces the isomorphism $\pi_{n}^{T[r]}(X, u) \simeq$ $\prod_{i=1}^{r} \underline{\pi}_{n}\left(X, u_{i}\right)$ if and only if $H$ is trivial.

Corollary 2.5. If $u$ : $T[r] \rightarrow \mathbb{R}^{k}$ is any base $T[r]$-tree

$$
\pi_{n}^{T[r]}\left(\mathbb{R}^{k}, u\right) \simeq \prod_{i=1}^{r} \pi_{n}\left(S^{k-1}\right)
$$

\section{Mobility}

Given two maps $u, v: S^{k} \rightarrow X$ and a path $\alpha$ running from $u(*)$ to $v(*)$ we say that $u$ and $v$ are $\alpha$-homotopic if there is a homotopy $H: S^{k} \times I \rightarrow X$ such that $H_{0}=u, H_{1}=v$ and $H(*, t)=\alpha(t)$.

Given $A, B \subset X$ we say $A$ is $(\alpha, n)$-movable to $B$ if each $u:\left(S^{n}, *\right) \rightarrow\left(A, \alpha_{0}\right)$ is $\alpha$-homotopic, in $X$, to a map $v:\left(S^{n}, *\right) \rightarrow\left(B, \alpha_{1}\right)$ where $\alpha_{0}=\alpha(0)$ and $\alpha_{1}=\alpha(1)$. If $i: A \rightarrow X$ and $j: B \rightarrow X$ are the canonical inclusions the above definition is equivalent to $\alpha_{\#} i_{*} \pi_{n}\left(A, \alpha_{0}\right) \subset j_{*} \pi_{n}\left(B, \alpha_{1}\right)$. where $\alpha_{\#}$ denotes the base point changing isomorphism associated to $\alpha$.

When $j_{*}(v)=\alpha_{\#} i_{*}(\mu)$ it is said that $\mu$ is $\alpha$-movable to $v$ and $\alpha$ is a path of mobility from $\mu$ to $v$.

We say $\alpha$ is not an $n$-mobility path from $A$ to $B$ if the only element of $\pi_{n}\left(A, \alpha_{0}\right)$ movable to $\pi_{n}\left(B, \alpha_{1}\right)$ is the unity. Otherwise we say $\alpha$ is a path of n-mobility. Finally $\alpha$ is a path of trivial $n$ mobility if each element of $\pi_{n}\left(A, \alpha_{0}\right)$ is $\alpha$-movable to each element of $\pi_{n}\left(B, \alpha_{1}\right)$.

\section{Remarks 3.1.}

(1) An element $\mu \in \pi_{n}\left(A, \alpha_{0}\right)$ can be $\alpha$-movable to several elements of $\pi_{n}\left(B, \alpha_{1}\right)$. If $A=B=S^{1}$ and $X$ is the cone over $A \Perp B$ then any path from $A$ to $B$ has trivial 1-mobility.

(2) If $\mu, \mu^{\prime} \in \pi_{n}\left(A, \alpha_{0}\right)$ are $\alpha$-movable to $B$ then $\mu+\mu^{\prime}$ (the product if $k=1$ ) is $\alpha$-movable to $B$.

(3) If $\alpha, \alpha^{\prime}: I \rightarrow X$ are homotopic paths, rel. $\{0,1\}$, then $\alpha$-mobility is equivalent to $\alpha^{\prime}$-mobility.

(4) The $\alpha$-mobility relation is not reflexive: If $A=A^{\prime}=S^{1}, X=A \vee A^{\prime}$ and $\{*\}=$ $A \cap A^{\prime}$, given generators $\mu \in \pi_{1}(A, *)$ and $v=[\alpha] \in \pi_{1}\left(A^{\prime}, *\right)$ then $\mu$ is not $v$-movable to itself.

(5) If $X, A$ and $B$ are path-connected it is a simple exercise to prove that any $\alpha:(I, 0,1) \rightarrow(X, A, B)$ is a path of trivial $n$-mobility if and only if $i_{*} \pi_{n}(A, a)=0=$ $j_{*} \pi_{n}(B, b)$. 
We next study the mobility between two components of the boundary of a compact surface.

Proposition 3.2. Let $S$ be a compact surface and $\alpha: I \rightarrow S$ such that $\alpha_{0}=\alpha(0)$ and $\alpha_{1}=\alpha(1)$ belong to $\partial S$. Then:

(a) If $\pi_{1}(S)$ is abelian and $S \neq B^{2}$, we can choose generators of $\pi_{1}\left(\partial S, \alpha_{0}\right)$ and $\pi_{1}\left(\partial S, \alpha_{1}\right)$ such that the only elements $\alpha$-movable are those which have the same multiplicity.

(b) If $S=B^{2}, \alpha$ is a path of trivial 1-mobility.

(c) If $\pi_{1}(S)$ is non-abelian and $\alpha_{0}$ and $\alpha_{1}$ are in two components $V_{0}$ and $V_{1}$ of $\partial S, \alpha$ is not an 1-mobility path.

(d) If $\pi_{1}(S)$ is non-abelian and $\alpha_{0}$ and $\alpha_{1}$ belong to the same component $V \subset \partial S$ then $\alpha$ is a path of 1-mobility if and only if it is homotopic, rel. $\partial S$, to a path $\alpha^{\prime}$ contained in $V$. In that case, there are generators of $\pi_{1}\left(V, \alpha_{0}\right)$ and $\pi_{1}\left(V, \alpha_{1}\right)$ such that the $\alpha$-movable elements are those which have the same multiplicity.

Proof.

(a) Since $\pi_{1}(S)$ is abelian, $S$ is homeomorphic to $S^{1} \times l$ or to the Moebius strip. If $\alpha_{0}$ and $\alpha_{1}$ belong to the same component $V \subset \partial S$, let $\varepsilon$ be an arc in $V$ which runs from $\alpha_{1}$ to $\alpha_{0}$.

It is easy to prove that $\mu \in \pi_{1}\left(V, \alpha_{0}\right)=\pi_{1}\left(V, \alpha_{1}\right)$ is $\alpha$-movable if and only if it is $\omega$-movable where $\omega=\varepsilon \circ \alpha$. But $\omega_{*}[\mu]=\omega^{-1} \circ \mu \circ \omega=\mu$.

If $\alpha_{0} \in V_{0}$ and $\alpha_{1} \in V_{1}$, where $V_{0}$ and $V_{1}$ are different components of $\partial S$, then $S$ is homeomorphic to $S^{1} \times I$. Given $\mu=[u] \in \pi_{1}\left(V_{0}, \alpha_{0}\right)$ and $\mu^{\prime}=\left[u^{\prime}\right] \in \pi_{1}\left(V_{1}, \alpha_{1}\right)$, Hurewicz's Theorem implies that $\left[\alpha^{-1} \circ u \circ \alpha\right]=\left[u^{\prime}\right]$ if and only if $\alpha^{-1} \circ u \circ \alpha$ and $u^{\prime}$ are homologous cycles. Therefore $\mu$ is $\alpha$-movable to $\mu^{\prime}$ if and only if they give the same homology class.

(b) It is obvious.

(c) Assume that $\partial S$ has more than two components. If $V_{0}$ and $V_{1}$ are two of such components and $\mu \in \pi_{1}\left(V_{0}, \alpha_{0}\right)$ is $\alpha$-movable to $\mu^{\prime} \in \pi_{1}\left(V_{1}, \alpha_{1}\right)$ then $\mu$ and $\mu^{\prime}$ have representatives which are homologous cycles. But this statement is in contradiction with the structure of $H_{1}(S)$.

If $\partial S$ has exactly two components then $S$ has the homotopy type of a wedge of three or more copies of $S^{1}$. Now, assuming the 1-mobility of $\alpha$ it is not hard to find a non-trivial relation in $\pi_{1}(S)$ which leads to contradiction.

(d) The proof uses the same arguments as the above cases.

Definition 3.3. Let $u: T[r] \rightarrow X$ be $p$-map and $u_{i}: \mathbb{R}_{+} \rightarrow X(1 \leqq i \leqq r)$ be the restriction to the $i$ th branch of $T[r]$. We say $\left[f_{i}\right] \in \underline{\pi}_{n}\left(X, u_{i}\right)$ is movable to $\left[f_{j}\right] \in \underline{\pi}_{n}\left(X, u_{j}\right)$ if there exists a natural number $m \in \mathbb{N}$ such that $\left[f_{i}^{\prime}\right] \in \pi_{n}\left(X, u_{i}\left(m_{i}\right)\right)$ is $u_{i j}(m)$-movable to 
$\left[f_{j}^{\prime}\right] \in \pi_{n}\left(X, u_{j}\left(m_{j}\right)\right)$ where $m_{i}$ and $m_{j}$ are the points of the branches $i$ and $j$ which have norm $m, f_{i}^{\prime}=f_{i}\left|S^{n} \times\left\{m_{i}\right\}, f_{j}^{\prime}=f_{j}\right| S^{n} \times\left\{m_{j}\right\}$ and $u_{i j}(m)=u \mid\left[0, m_{i}\right] \cup\left[0, m_{j}\right]$.

Remark 3.4. If $\left[f_{i}\right]$ is movable to $\left[f_{j}\right]$ in $(X, u)$ then $f_{i} \mid S^{n} \times\left\{m_{i}\right\}$ is $u_{i j}(m)$-movable to $f_{j} \mid S^{n} \times\left\{m_{j}\right\}$ for any $m \in \mathbb{N}$.

The following results are only restatements of those of Section 2. We give them without proof.

Proposition 3.5. With the notation of Theorem 2.1, given $\left[f_{i}\right] \in \pi_{n}\left(X, u_{i}\right)(1 \leqq i \leqq r)$, $\left(\left[f_{1}\right],\left[f_{2}\right], \ldots,\left[f_{r}\right]\right) \in G$ if and only if $\left[f_{i}\right]$ is movable to $\left[f_{j}\right]$ in $(X, u)$ for each pair $i, j$.

Corollary 3.6. With the hypotheses of Corollary 2.4 we have:

(a) $\pi_{n}^{T[r]}(X, u)$ is in 1-1 correspondence with the elements

$$
\left(\left[f_{1}\right],\left[f_{2}\right], \ldots,\left[f_{r}\right]\right) \in \prod_{i=1}^{r} \pi_{n}\left(X, u_{i}\right)
$$

such that $\left[f_{i}\right]$ is movable to $\left[f_{j}\right]$ in $(X, u)$ for each pair $i, j$.

(b) $H=1$ if and only if, given $\alpha_{i} \in \pi_{n}\left(X, u_{i}\right)$ and $\alpha_{j} \in \pi_{n}\left(X, u_{j}\right), \alpha_{i}$ is movable to $\alpha_{j}$ in $(X, u)$.

Corollary 3.7. Let $S$ be a compact surface, $x_{i} \in S-\partial S(1 \leqq i \leqq r)$ and $X=S-\left\{x_{1}, \ldots, x_{s}\right\}$ Then:

(a) $\pi_{n}^{T[r]}(X, u)=0$ if $n \geqq 2$.

(b) If $\pi_{1}\left(X, x_{0}\right)$ is abelian, $\pi_{1}^{T[r]}(X, u) \simeq \mathbb{Z}$ when $X \neq \mathbb{R}^{2}$. If $X=\mathbb{R}^{2}, \pi_{1}^{T[r]}(X, u) \simeq \bigoplus^{r} \mathbb{Z}$.

(c) If $\pi_{1}\left(X, x_{0}\right)$ is non-abelian we have

$\left(c_{1}\right) \pi_{1}^{T(r)}(X, u) \simeq \mathbb{Z}$ if all the branches of $T[r]$ define the same proper end of $X$ and there is a p-homotopy which carries $u$ into a neighbourhood of that end.

(c $\left.\mathrm{c}_{2}\right) \pi_{1}^{T[r]}(X, u)=1$ otherwise.

Proof. As $\pi_{n+1}\left(X, x_{0}\right)=0$ if $n \geqq 1$, Theorem 2.1 says that $\pi_{n}^{T[r]}(X, u)$ is the pull-back of the Cerin-groups $\underline{\pi}_{n}\left(X, u_{i}\right)$. In [7] it is shown an isomorphism $\Psi: \underline{\pi}_{n}\left(X, u_{i}\right) \rightarrow \pi_{n}\left(F r U_{i}\right)$, where $\operatorname{Fr} U_{i}$ is the boundary of a cylindrical neighbourhood $U_{i}$ of the end defined by $u_{i}$. Now (a) follows easily.

By Corollary 3.6, each element of $\pi_{1}^{T(r)}(X, u)$ is uniquely determined by an element

$$
\left(\left[f_{1}\right],\left[f_{2}\right], \ldots,\left[f_{r}\right]\right) \in \prod_{i=1}^{r} \pi_{1}\left(X, u_{i}\right)
$$

such that $\left[f_{i}\right]$ is movable to $\left[f_{j}\right]$ for each pair $i, j$. Let $\varepsilon(i)$ be the proper end defined by $u_{i}$ and let $U_{i}$ be a cylindrical neighbourhood of $\varepsilon(i)$ such that $f_{i}\left(S^{1} \times[m, \infty)\right) \subset U_{i}$ where 
$m$ is sufficiently large. If $p_{i}: U_{i} \rightarrow \operatorname{Fr} U_{i}$ is the natural projection, the isomorphism $\Psi$ of [7] is given by $\Psi\left(\left[p_{i}\right]\right)=\left[p_{i} \circ f_{i}\right]$ and it is easy to prove that $p_{i} \circ f_{i} \mid S^{1} \times\{m\}$ is $\alpha$-movable to $p_{j} \circ f_{j} \mid S^{1} \times\{m\}$ where $\alpha$ is the path $u \mid\left[0, m_{i}\right] \cup\left[0, m_{j}\right]$ deformed by $p_{i}$ and $p_{j}$. Then (b) and (c) follow from Proposition 3.2 applied to $S-\bigcup_{i=1}^{r} U_{i}$.

The result from [7] used in the proof of the previous corollary can be applied again to prove the following lemma.

Lemma 3.8. Let $X$ be the complement of an interior point of a compact surface distinct from $S^{2}$. Then the canonical restriction

$$
\rho: \underline{\pi}_{1}(X, w) \rightarrow \pi_{1}\left(X, x_{0}\right)
$$

is injective for any ray $w: \mathbb{R}_{+} \rightarrow X$.

Corollary 3.9. If the extra hypothesis

$$
\partial S \cap\left\{x_{1}, \ldots, x_{s}\right\}=\left\{x_{1}, \ldots, x_{q}\right\}
$$

is added to Corollary 3.7 then

(a) $\pi_{n}^{T[r]}(X, u)=0(n \geqq 2)$.

(b) If the branches of $T[r]$ miss the ends defined by $\left\{x_{1}, \ldots, x_{q}\right\}$ then $\pi_{1}^{T[r]}(X, u) \simeq$ $\pi_{1}^{T(r)}\left(X^{\prime}, u\right)$ where $X^{\prime}=S-\left\{x_{q+1}, \ldots, x_{s}\right\}$ and then it follows as Corollary 3.7.

(c) Otherwise $\pi_{1}^{T[r]}(X, u)=1$.

Proof (a) and (b) follow as in Corollary $3.7\left(\pi_{1}(X)=\pi_{1}\left(X^{\prime}\right)\right)$.

(c) If $u_{i_{0}}$ goes towards $x_{j 0} \in \partial S$ it is easy to prove (using again [7]) $\pi_{1}\left(X, u_{i_{0}}\right)=1$. Then Theorem 2.1 gives $\pi_{1}^{T[r]}(X, u) \simeq \prod\left\{\operatorname{Ker} \rho_{i} ; i \in \Lambda\right\}$ where $\Lambda$ is the set of branches which go towards points of $\left\{x_{q+1}, \ldots, x_{s}\right\}$. We conclude by applying Lemma 3.8.

\section{Product theorem}

In this section the spaces are Hausdorff and $T$ is $T[r, k]$.

Theorem 4.1. Let $\left(X, x_{0}\right)$ be a based compact space. Given a noncompact space $Y$ and a T-tree $u^{\prime}: T \rightarrow Y$ there is a natural bijection (isomorphism if $n \geqq 1$ ).

$$
\varphi: \pi_{n}^{T}(X \times Y, u) \rightarrow \pi_{n}\left(X, x_{0}\right) \times \pi_{n}^{T}\left(Y, u^{\prime}\right) \quad(n \geqq 0)
$$

where $u: T \rightarrow X \times Y$ is $u(z)=\left(x_{0}, u^{\prime}(z)\right)$.

Proof. Given $f: S^{n} \times T \rightarrow X \times Y$ we define

$$
\varphi[f]=\left(\left[f_{1} \circ j_{1}\right],\left[f_{2}\right]\right) \in \pi_{n}\left(X, x_{0}\right) \times \pi_{n}^{T}\left(Y, u^{\prime}\right)
$$

where $p_{1}: X \times Y \rightarrow X$ and $p_{2}: X \times Y \rightarrow Y$ are the natural projections, $j: S^{n} \rightarrow S^{n} \times T$ is $j(z)=\left(z, v_{0}\right)$ and $f_{i}=p_{i} \circ f(i=1,2)$. It is easy to prove that is an isomorphism if $n \geqq 1$. 
On the other hand if $\left(\left[h_{1}\right],\left[h_{2}\right]\right) \in \pi_{n}\left(X, x_{0}\right) \times \pi_{n}^{T}\left(Y, u^{\prime}\right)$ we take the $p$-map $h: S^{n} \times T \rightarrow$ $X \times Y$ given by $h(z, v)=\left(h_{1}(z), h_{2}(z, v)\right)$. Then $\varphi([h])=\left(\left[h_{1}\right],\left[h_{2}\right]\right)$, so $\varphi$ is epijective.

Finally, if $\varphi([f])=\varphi\left(\left[f^{\prime}\right]\right)$ there are homotopies $H_{1}: S^{n} \times\left\{v_{0}\right\} \times I \rightarrow X$ and $H_{2}: S^{n} \times T \times$ $I \rightarrow Y\left(H_{2}\right.$ proper $)$ such that

$$
\begin{gathered}
H_{1}\left(z, v_{0}, 0\right)=f_{1} \circ j(z) ; H_{1}\left(z, v_{0}, 1\right)=f_{1}^{\prime} \circ j(z) ; H_{1}\left(*, v_{0}, s\right)=x_{0} \\
H_{2}\left(z, v_{0}, 0\right)=f_{2}(z, v) ; H_{2}(z, v, 1)=f_{2}^{\prime}(z, v) ; H_{2}(*, v, s)=u^{\prime}(v) .
\end{gathered}
$$

These conditions allow us to define the map

$$
H_{1}^{\prime}=f_{1} \cup f_{1}^{\prime} \cup H_{1}: S^{n} \times\left(T \times\{0,1\} \cup\left\{x_{0}\right\} \times I\right) \rightarrow X .
$$

By the H.E.P. we can extend $H_{1}^{\prime}$ to $\tilde{H}_{1}: S^{n} \times T \times I \rightarrow X$. Then

$$
H=\left(\widetilde{H}_{1}, H\right): S^{n} \times T \times I \rightarrow X \times Y
$$

is a homotopy between $f_{1}$ and $f_{2}$. Notice that $H$ is proper: For each compactum $K \subset Y$, $H^{-1}(X \times K) \subset H_{2}^{-1}(K)$.

Corollary 4.2. Let $X$ be compact and path-connected and $Y$ noncompact.

(a) Then $\left[\mathbb{R}_{+} ; Y\right]_{p} \simeq\left[\mathbb{R}_{+} ; X \times Y\right]_{p}\left([f] \in\left[\mathbb{R}_{+} ; Y\right]_{p}\right.$ is called a proper end of $Y$. See $[6])$.

(b) If $u: T \rightarrow X \times Y$ is any T-tree and $u^{\prime}=p_{2} \circ u$,

$$
\pi_{n}^{T}(X \times Y, u) \simeq \pi_{n}\left(X, x_{0}\right) \times \pi_{n}^{T}\left(Y, u^{\prime}\right) .
$$

\section{Proof.}

(a) It follows from Theorem 4.1 with $n=0, T=\mathbb{R}_{+}$.

(b) The proof of Theorem 4.1 with $n=0$ shows that $u$ and $\left\{x_{0}\right\} \times u^{\prime}$ are $p$-homotopic maps. Now it follows from [1; Theorem 2].

Remark 4.3. The following examples show that there is no generalization of Theorem 4.1 in a natural way when $X$ is noncompact. Given $(\mathbb{R}, i d)$ we can consider $\left(\mathbb{R}^{2}, u\right)$ and $\left(\mathbb{R}^{2}, u^{\prime}\right)$ where $u=i d \times i d$ and $u^{\prime}=0 \times i d$. Then, from Corollary 1.4 and Remark $1.6, \pi_{1}^{T[2.0]}(\mathbb{R}, u)=1, \pi_{1}^{T[2,1]}\left(\mathbb{R}^{2}, u\right) \simeq \mathbb{Z}$ and $\pi_{1}^{T[2,0]}\left(\mathbb{R}^{2}, u^{\prime}\right) \simeq \mathbb{Z} \oplus \mathbb{Z}$.

In spite of the above remark, the following product theorem involving noncompact spaces can be proved.

Theorem 4.4. Let $\left(X, x_{0}\right)$ be a based space, compact or not. If $u: \mathbb{R}_{+} \rightarrow X \times \mathbb{R}_{+}$is $u(t)=\left(x_{0}, t\right)$ then $\underline{\pi}_{n}\left(X \times \mathbb{R}_{+} u\right) \simeq \pi_{n}\left(X, x_{0}\right),(n \geqq 1)$.

Proof. Given $f: S^{n} \times \mathbb{R}_{+} \rightarrow X \times \mathbb{R}_{+}$we denote by $f_{0}$ the restriction $p_{1} \circ f \mid S^{n} \times\{0\}$ : $S^{n} \rightarrow X$ where $p_{1}: X \times \mathbb{R}_{+} \rightarrow X$ is the natural projection. It is easy to prove that 


$$
\Psi: \underline{\pi}_{n}\left(X \times \mathbb{R}_{+}, u\right) \rightarrow \pi_{n}\left(X, x_{0}\right)
$$

given by $\Psi([f])=\left[f_{0}\right]$ is an epimorphism.

In order to prove that $\Psi$ is injective, we assume that $\Psi([f])=\left[f_{0}\right]=\left[g_{0}\right]=\Psi([g])$. Then there is a homotopy $F$ between $f_{0}$ and $g_{0}$. The new map

$$
\tilde{F}: S^{n} \times \mathbb{R}_{+} \times I \rightarrow X \times \mathbb{R}_{+}
$$

given by $\tilde{F}(z, s, t)=(F(z, t), s)$ is a $p$-homotopy, rel. $u$, between $\tilde{f}_{0}$ and $\tilde{g}_{0}$ where $\tilde{f}_{0}, \tilde{g}_{0}: S^{n} \times \mathbb{R}_{+} \rightarrow X \times \mathbb{R}_{+}$are given by $\tilde{f}_{0}(z, s)=\left(f_{0}(z), s\right)$ and $\tilde{g}_{0}(z, s)=\left(g_{0}(z), s\right)$. It suffices to prove that $\tilde{f}_{0}$ and $\tilde{g}_{0}$ are $p$-homotopic, rel. $u$, to $f$ and $g$ respectively. We will find the $p$-homotopy between $\tilde{f}_{0}$ and $f$. The other $p$-homotopy is analogous.

Firstly, we consider the map $H: S^{n} \times \mathbb{R}_{+} \times I \rightarrow X \times \mathbb{R}_{+}$given by

$$
H(z, s, t)=\left(p_{1} \circ f(z, s), t s+(1-t) p_{2} \circ f(z, s)\right) .
$$

$H$ is a homotopy, rel. $u$, between $f$ and $f(z, s)=\left(p_{1} \circ f(z, s), s\right)$.

In fact $H$ is a $p$-homotopy: Let $K \subset X$ be a compactum. Given any $s_{0} \in \mathbb{R}_{+}$and a net $\left\{\left(z_{\delta}\right.\right.$ we need to find a cluster point in this set. But $\left\{\left(z_{\delta} t_{\delta}\right)\right\} \subset S^{n} \times I$ so it suffices to give a cluster point of $\left\{s_{\delta}\right\}$.

We know that $\left\{p_{1} \circ f\left(z_{\delta} s_{\delta}\right)\right\} \subset K$ and

$$
t_{\delta} s_{\delta}<t_{\delta} s_{\delta}+\left(1-t_{\delta}\right) p_{2} \circ f\left(z_{\delta}, s_{\delta}\right) \leqq s_{0} .
$$

Then we can assume that $\lim t_{\delta} s_{\delta}$ exists. If $\lim t_{\delta} \neq 0$ then $\lim s_{\delta}$ exists and it is a cluster point. Only the case $\lim t_{\delta}=0$ remains. But then

$$
\lim p_{2} \circ f\left(z_{\delta}, s_{\delta}\right) \leqq \lim \left\{t_{\delta} s_{\delta}+\left(1-t_{\delta}\right) p_{2} \circ f\left(z_{\delta}, s_{\delta}\right)\right\} \leqq s_{0}
$$

and there exists $\delta_{0}$ such that $p_{2} \circ f\left(z_{\delta}, s_{\delta}\right) \in\left[0, s_{0}+1\right]$ for any $\delta \geqq \delta_{0}$. So $f\left(z_{\delta}, s_{\delta}\right) \in K \times$ $\left[0, s_{0}+1\right]$ for any $\delta \geqq \delta_{0}$ and $\left\{s_{\delta}\right\}$ has a cluster point because $f$ is a proper map.

Finally, we define

$$
G: S^{n} \times \mathbb{R}_{+} \times I \rightarrow X \times \mathbb{R}_{+}
$$

by $G(z, s, t)=\left(p_{1} \circ f(z, t s), s\right)$. Then $G$ is a homotopy between $\tilde{f}$ and $\tilde{f}_{0}$, rel. $u$, and it a $p$-map because

$$
G^{-1}\left(K \times\left[0, s_{0}\right]\right) \subset S^{n} \times\left[0, s_{0}\right] \times I
$$

for any compactum $K \subset Y$ and $s_{0} \in \mathbb{R}_{+}$.

\section{Remarks 4.5.}

(a) Theorem 4.4 fails if a general tree replaces $\mathbb{R}_{+}$(see Remark 4.3 ). 
(b) Theorem 4.4 with $X$ compact is proved in [7].

(c) For $n=0$, it is proved in [6] that if $X$ and $Y$ are noncompact $T_{2}$-locally $\sigma$-compact and path-connected then $X \times Y$ has only one proper end.

When $Y=\mathbb{R}_{+}$the topological conditions on $X$ can be dropped and we can obtain the following particular result.

Theorem 4.6. Let $X$ be a path-connected space. Then any two p-maps $\alpha, \beta: \mathbb{R}_{+} \rightarrow$ $X \times \mathbb{R}_{+}$are p-homotopic.

Proof. Let $p_{1}: X \times \mathbb{R}_{+} \rightarrow X$ and $p_{2}: X \times \mathbb{R}_{+} \rightarrow \mathbb{R}_{+}$be the natural projections. Given the points $a=p_{1} \alpha(0)$ and $b=p_{1} \beta(0)$, define the $p$-maps

$$
\tilde{\alpha}_{0}, \tilde{\beta}_{0}, \tilde{\alpha}, \tilde{\beta}: \mathbb{R}_{+} \rightarrow X \times \mathbb{R}_{+}
$$

by the formulae

$$
\begin{gathered}
\tilde{\alpha}_{0}(v)=(a, v) ; \tilde{\beta}_{0}(v)=(b, v) ; \\
\tilde{\alpha}(v)=\left(p_{1} \alpha(v), v\right) ; \tilde{\beta}(v)=\left(p_{1} \beta(v), v\right) .
\end{gathered}
$$

To prove the theorem it suffices to show that there are $p$-homotopies $\alpha \sim \tilde{\alpha} \sim \tilde{\alpha}_{0} \sim \tilde{\beta}_{0} \sim$ $\tilde{\beta}$. By using the same arguments as the above theorem, it can be seen that $H$ : $\mathbb{R}_{+} \times I \rightarrow X \times \mathbb{B}_{+}$defined by

$$
H(v, t)=\left(p_{1} \alpha(v), t v+(1-t) p_{2} \alpha(v)\right)
$$

is a p-homotopy from $\alpha$ to $\tilde{\alpha}$. Similarly, $G(v, t)=\left(p_{1} \alpha(t v), v\right)$ is a $p$-homotopy between $\tilde{\alpha}$ and $\tilde{\alpha}_{0}$. But if $\mu: I \rightarrow X$ is a path from $a$ to $b$, it is obvious that $F(v, t)=(\mu(v), t)$ determines a $p$-homotopy from $\tilde{\alpha}_{0}$ to $\widetilde{\beta}_{0}$, and this completes the proof.

Remark 4.7. Theorem 4.6 fails if the $T_{2}$ condition is dropped: if $X=[0, \infty)$ with the indiscrete topology, $\pi_{0}\left(X \times \mathbb{R}_{+}\right)$has the continuum cardinality and $\pi_{0}\left(X, x_{0}\right)$ is trivial.

Acknowledgement. This work was supported by CAICYT grant $0812-84$.

\section{REFERENCES}

1. R. Ayala, E. Dominguez, A. Quintero, Cylindrical $p$-homotopy groups, preprint.

2. H. J. Baues, Algebraic homotopy (Cambridge Studies in Advanced Mathematics, 15, 1987).

3. M. Brin and T. L. Thickstun, On the proper Steenrod homotopy groups and proper embeddings of planes into 3 manifolds, Trans. Amer. Math. Soc. 289 (1985), 737-755.

4. E. M. Brown, On the proper homotopy type (Lecture Notes Mathematics 375, SpringerVerlag, 1974). 
5. Z. ČRIN, On various relative proper homotopy groups, Tsukuba J. Math. 4 (1980) 177-202.

6. E. Dominguez, L. J. Hernández, Remarks about proper ends, preprint.

7. E. Dominguez, L. J. Hernández, Unas notas sobre los finales de un espacio, preprint.

8. D. A. Edwards, H. M. Hastings, Cech and Steenrod homotopy theories with applications to Geometric Topology (Lectures Notes Mathematics 542, Springer-Verlag, 1976).

9. J. R. JACKson, On homotopy groups of function spaces, Amer. J. Math. 74 (1952), 241-252.

10. S. S. KoH, Note on the homotopy properties of the components of the mapping space $X^{s p}$, Proc. Amer. Math. Soc. 11 (1960), 896-904.

11. C. R. F. MAunder, Algebraic Topology (Van Nostrand, 1970).

12. T. Porter, Cech and Steenrod homotopy and the Quigley exact couple in strong shape and proper homotopy theory, J. Pure Appl. Alg. 24 (1983), 303-312.

13. T. Porter, Homotopy groups for strong shape and proper homotopy, Convegno di Topologia. Suppl. Rend. Circolo Mat. Palermo 4 (1984), 101-113.

14. E. Spanier, Algebraic Topology (McGraw-Hill, 1966).

Departamento de Geometria y Topología

Facultad de Matemáticas

Universidad de Sevilla

41012 SeVILla

SPAIN
Departamento de Matemáticas Facultad de Ciencias UNIVERSIDAD DE ZARAGOZA 50009 ZaraGozA

SPain 